\title{
The Impact of the Liberal Use of CT in the Work Up of Acute Appendicitis
}

\author{
Omar H. Llaguna, Dimitrios Avgerinos, Andrew Cha, Richard Friedman, Burton G. Surick and \\ I. Michael Leitman
}

Department of Surgery, Albert Einstein College of Medicine - Beth Israel Medical Center, New York, NY, USA

\begin{abstract}
Background: Acute appendicitis has historically been considered a clinical diagnosis, necessitating a CT scan only in the face of equivocal signs and symptoms. The purpose of this study is to determine whether or not the liberal use of CT in the work up of acute appendicitis results in a decreased negative appendectomy rate (NAR).

Methods: The medical records of 940 consecutive patients who underwent emergency appendectomy between January 2002 and December 2006 were reviewed. Data collected included patient demographics, length of stay (LOS), results of contrast enhanced CT scans, emergency department (ED) work up time, and final pathology.

Results: During the study period 940 patients (mean age $34.5 \pm 16.7$ years, 53\% males) underwent emergency appendectomy (64\% laparoscopic). Eight hundred thirteen (86\% patients, 50\% males) underwent pre-operative CT scans. Final pathology was $76.1 \%$ acute appendicitis, $10 \%$ acute appendicitis with perforation, $7.7 \%$ gangrenous appendicitis, $1.2 \%$ acute appendicitis with abscess, $0.7 \%$ chronic appendicitis, and $4.3 \%$ negative for appendicitis. No difference was noted in the overall appendicitis rate by gender in the CT (Fishers exact test, $\mathrm{p}=0.96$ ) or non-CT group (Fishers exact test, $\mathrm{p}=0.75)$. Similarly, no difference was noted by age $(\mathrm{CT} p=0.14$, non-CT $\mathrm{p}=0.26)$. The NAR was lower for the CT group $(3.4 \%)$ compared to the non-CT group $(9.4 \%)(\mathrm{p}=0.002)$. The NAR did not differ significantly by gender (Fishers exact test, $\mathrm{p}=0.491)$. Despite prolonging the ED work up time by 4.3 hours, the perforation rate did not differ between the CT $(9.5 \%)$ and non-CT $(12.6 \%)$ groups $(p=0.30)$, nor did the LOS (3.67 vs. 3.63 days, $p=0.92)$. The sensitivity, specificity, negative predictive value, and positive predictive value of CT scans was $99 \%, 61 \%, 68 \%$, and $99 \%$, with an overall accuracy of $98 \%$. The positive and negative likelihood ratios were 2.52 and 0.02 respectively. The ROC for CT scans in predicting appendicitis was $79.8 \%$ (95\% CI: 76.9\%, 82.5\%).

Conclusions: The use of CT in the work up of acute appendicitis is associated with a lower negative appendectomy rate independent of gender and age. The additional time spent obtaining a CT does not appear to increase the risk of perforation nor increase the LOS.
\end{abstract}

\section{BACKGROUND}

Despite sound clinical judgment and the availability of modern imaging modalities, negative appendectomy rates as high as $15 \%$ for the general population and $23 \%$ to $35 \%$ for women are still reported [1]. These high rates have traditionally been accepted by surgeons, comforted by a belief that the morbidity and the costs of a missed appendicitis greatly outweigh that of a negative appendectomy. Large population based studies however, disagree with this benign outlook reporting considerable clinical and economic cost [1]. For this reason, in an attempt to improve on the traditionally tolerated negative appendectomy rate, much attention has been turned towards redesigning the work up of acute appendicitis.

Acute appendicitis has historically been considered a clinical diagnosis, necessitating a CT scan only in the face of equivocal signs and symptoms. However, since its advent clinicians have struggled to expand the role of CT in the work up of right lower quadrant pain. Some recommend a selective approach justifying its use in specific age groups,

*Address correspondence to this author at the Department of Surgery, Albert Einstein College of Medicine - Beth Israel Medical Center, 10 Union Square East, Suite 2M, New York, NY 10003, USA;

E-mail: MLeitman@chpnet.org women of reproductive age, and in those with an equivocal presentation [2-6]. Others advocate its routine use citing $90 \%$ to $100 \%$ sensitivity, $91 \%$ to $99 \%$ specificity, and a positive predictive value of $95 \%$ to $97 \%$ [7]. Proponents of routine CT argue that it improves on the traditionally accepted $15 \%$ to $20 \%$ negative appendectomy rate without any additional morbidity [8-15]. Still, another subset of clinicians fail to report any clear benefit from either selective or routine strategies, believing it does not significantly decrease negative appendectomy rates and may furthermore negatively impact outcomes by delaying definitive management [1619].

Most medical centers do not have an established protocol for the work up of acute appendicitis. However, an increasingly tolerant attitude exists among surgeons and emergency medicine physicians regarding the acquisition of a CT scan during the work up of acute right lower quadrant pain. In order to assess the influence of CT on our institution's negative appendectomy rate, a retrospective chart review was performed of all patients greater than age 13 who underwent appendectomy from January 2002 to December 2006. The purpose of the study was to determine whether or not the liberal use of CT in the work up of acute appendicitis significantly reduces the negative appendectomy rate and whether it negatively influenced perforation rates. 
Table 1. Demographics and Pathologic Data

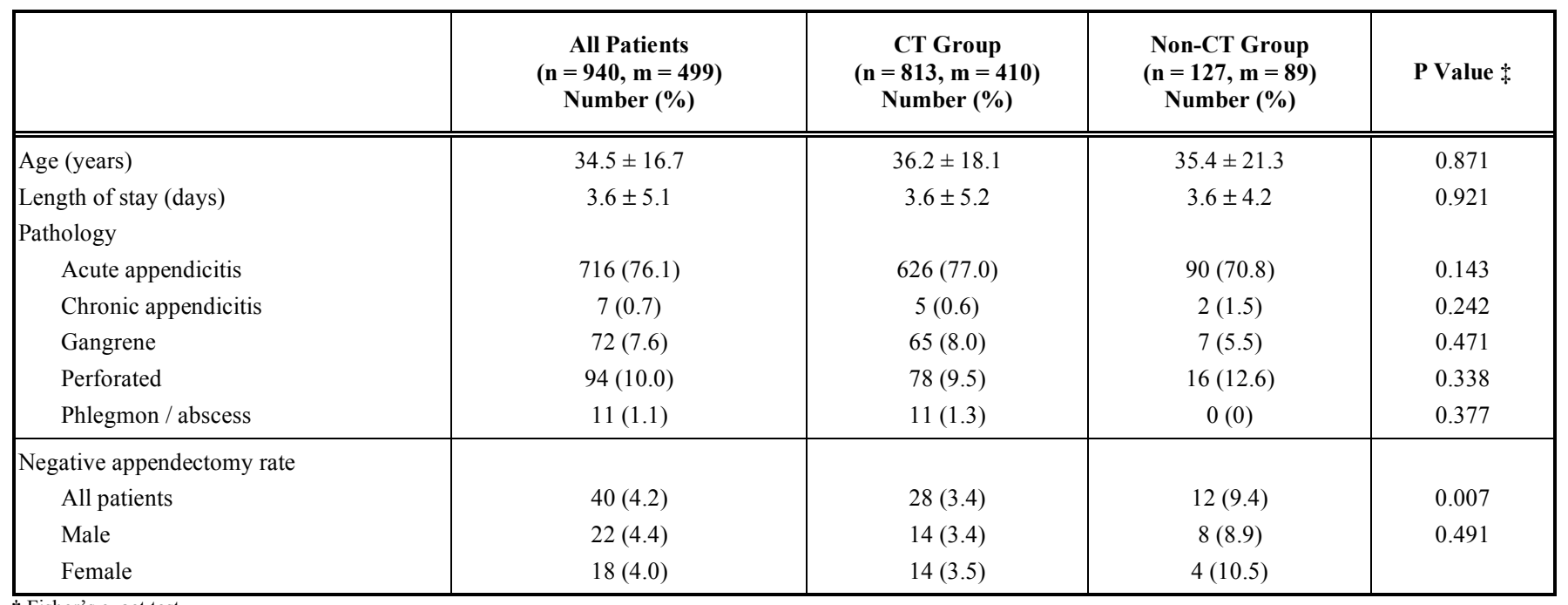

$\$$ Fisher's exact test.

\section{PATIENTS AND METHODS}

After obtaining approval from the Beth Israel Medical Center Institutional Review Board (IRB \# 103-07) the medical records of 940 consecutive patients above age 13 who underwent emergency appendectomy for presumed acute appendicitis between January 2002 and December 2006 where retrospectively reviewed. CT scan of the abdomen and pelvis was performed with oral contrast only. Intravenous contrast was only administered in patients in whom inflammation around the appendix was determined to be equivocal by a radiologist. The $\mathrm{Ct}$ was immediately reviewed by a radiology resident and within 1 hour by an attending radiologist. Patients with clear right lower quadrant peritoneal signs did not undergo CT scan, but rather were transferred immediately to the operating room for appendectomy. All female patients of childbearing age underwent pelvic examination also. Data collected included patient demographics (age and gender), procedure type (open versus laparoscopic), length of stay, results of contrast enhanced CT scans, CT work up time, and final pathology. Data was analyzed using Statistical Package for the Social Sciences for Windows 16 (SPSS Inc. Chicago, IL). A p value less than 0.05 was considered statistically significant.

\section{RESULTS}

During the study period 940 patients (53\% male, $47 \%$ female) underwent emergency appendectomy (70\% completed laparoscopically) for suspected acute appendicitis. The mean age of the group was $34.5 \pm 16.7$ (range 13 to 95, CT $36.2 \pm 18.1$ vs non-CT $35.4 \pm 21.3, \mathrm{p}=0.87$ ), eight hundred thirteen ( $86 \%$ patients, $50 \%$ males) of which underwent pre-operative CT scans.

For the cohort, final pathology was $76.1 \%$ acute appendicitis, $10 \%$ acute appendicitis with perforation, $7.6 \%$ gangrenous appendicitis, $1.1 \%$ acute appendicitis with abscess, $0.7 \%$ chronic appendicitis, and $4.2 \%$ negative for appendicitis. No statistical significance was noted between the CT and non-CT group for acute appendicitis $(\mathrm{p}=0.14)$, acute appendicitis with perforation $(\mathrm{p}=0.33)$, gangrenous appendicitis $(\mathrm{p}=0.47)$, acute appendicitis with abscess $(\mathrm{p}=0.37)$, and chronic appendicitis $(p=0.24)$. The overall negative appendectomy rate was lower for the CT group (3.4\%) compared to the non-CT group $(9.4 \%)(\mathrm{p}=0.007)$, and did not differ significantly by gender (Fishers exact test, $p=0.491$ ). (Table 1) No difference was noted in the overall negative appendectomy rate by gender in the CT (Fishers exact test, $\mathrm{p}=1.00$ ) or non-CT group (Fishers exact test, $\mathrm{p}=0.75$ ). Similarly, no difference was noted by age (CT p $=0.14$, non-CT, $\mathrm{p}=$ 0.26 ). (Tables 2 and 3) Despite the additional 4.3 hours required to obtain a CT scan, the perforation rate did not differ between the CT and non-CT group (9.5\% vs $12.6 \%, \mathrm{p}=$ 0.33 ), nor did the length of stay (3.67 vs 3.63 days, $\mathrm{p}=0.92$ ).

No statistically significant difference was noted in the number of CT scans obtained per year (Fishers exact test, $p=$ 0.36). (Table 4) The sensitivity, specificity, negative predictive value, and positive predictive value of CT scans was $99 \%, 61 \%, 68 \%$, and $99 \%$, with an overall accuracy of $98 \%$. The positive and negative likelihood ratios were 2.52 and 0.02 respectively (Table 5).

Table 2. Negative Appendectomy Rate by Age and Gender in the Non-CT Group

\begin{tabular}{|c|c|c|c|}
\hline & $\begin{array}{c}\text { Positive } \\
\text { Appendectomy } \\
\text { (n=115) } \\
\text { Number (\%) }\end{array}$ & $\begin{array}{c}\text { Negative } \\
\text { Appendectomy } \\
\text { (n= 12) } \\
\text { Number (\%) }\end{array}$ & P Value \\
\hline \hline $\begin{array}{c}\text { Age (years) } \\
\leq 16\end{array}$ & $22(19.6)$ & $4(36.4)$ & $0.256 \dagger$ \\
$17-30$ & $43(38.4)$ & $1(9.1)$ & \\
$31-45$ & $25(22.3)$ & $2(18.2)$ & \\
$46-60$ & $13(11.6)$ & $2(18.2)$ & \\
$\geq 61$ & $9(8.0)$ & $2(18.2)$ & \\
\hline Gender & $81(70.4)$ & $8(66.7)$ & $0.751 \ddagger$ \\
Male & $34(29.6)$ & $4(33.3)$ & \\
Female & & & \\
\hline
\end{tabular}

\footnotetext{
$\dagger$ Chi-square test; $\$$ Fisher’s exact test.
} 
Table 3. Negative Appendicitis Rate by Age and Gender in the CT Group

\begin{tabular}{|l|c|c|c|}
\hline & $\begin{array}{c}\text { Positive } \\
\text { Appendectomy } \\
(\mathbf{n = 7 8 5 )} \\
\text { Number (\%) }\end{array}$ & $\begin{array}{c}\text { Negative } \\
\text { Appendectomy } \\
\text { (n= 28) } \\
\text { Number (\%) }\end{array}$ & P Value \\
\hline \hline $\begin{array}{c}\text { Age (years) } \\
\leq 16\end{array}$ & $68(8.7)$ & $0(0)$ & $0.139 \dagger$ \\
$17-30$ & $301(38.5)$ & $8(29.6)$ & \\
$31-45$ & $237(30.3)$ & $10(37.0)$ & \\
$46-60$ & $115(14.7)$ & $4(14.8)$ & \\
$\geq 61$ & $61(7.8)$ & $5(18.5)$ & $1.000 \ddagger$ \\
Gender & $396(50.4)$ & $14(50.0)$ & \\
Male & $389(49.6)$ & $14(50.0)$ & \\
Female &
\end{tabular}

$\dagger$ Chi-square test; $\div$ Fisher's exact test.

Table 4. Number of CT Scans Obtained Per Year

\begin{tabular}{|c|c|c|c|c|}
\hline Year & CT & No CT & Total & P Value * \\
\hline \hline 2002 & 139 & 28 & 167 & $\mathrm{p}=0.36$ \\
2003 & 156 & 28 & 184 & \\
2004 & 175 & 26 & 201 & \\
2005 & 162 & 26 & 188 & \\
2006 & 181 & 19 & 200 & \\
\hline
\end{tabular}

\$ Fisher's exact test.

\section{Table 5. Validity of CT}

\begin{tabular}{|l|l|}
\hline Sensitivity & 0.99 \\
Specificity & 0.61 \\
Positive Predictive Value & 0.99 \\
Negative Predictive Value & 0.68 \\
Likelihood Ratio + & 2.52 \\
Likelihood Ratio - & 0.02 \\
False Positive Rate & 0.39 \\
False Negative Rate & 0.01 \\
Probability or Prevalence of disease & 0.97 \\
Overall Accuracy & 0.98 \\
\hline
\end{tabular}

\section{DISCUSSION}

Although the validity of CT in the diagnosis of acute appendicitis is well established $[3,4,7]$ its appropriate utilization continues to be a topic of debate. Should CT be used mandatorily, selectively, or not at all in the work up of acute appendicitis? A review of the literature reveals inconsistent and sometimes conflicting data. While many previous studies report an improved overall negative appendectomy rates with the use of CT, most find the benefit limited to a specific age group or gender, while few report no benefit at all. Despite not having an established protocol for the work up of acute appendicitis, $86 \%$ of our 940 patients received a CT scan. The overall negative appendectomy rate at our institution over the 5 year study period was $4.2 \%$, a rate considerably lower than the traditionally accepted 15 to $20 \%$. The negative appendectomy rate was significantly lower for the CT group compared to the non-CT group (3.4\% vs $9.4 \%$, $\mathrm{p}=0.007)$. Studies with similar findings are abundant. A multicenter retrospective review by $\mathrm{Kim}$, et al. revealed an inverse relationship (rho $=-1$ ) between the utilization of CT and the overall negative appendectomy rate $(20.6 \%$ vs $6.6 \%$, $\mathrm{p}<0.05)$ [12]. Guss, et al. reported a decrease in the overall negative appendectomy rate $(15.5 \%$ vs $7.9 \%, \mathrm{p}=0.001)$ following the implementation of a protocol which included CT [13]. Jones et al. also found a correlation between increased utilization of CT (52\% in $2000,74 \%$ in 2001 and $86 \%$ in 2002) and progressively lower negative appendectomy rates (17\% in $2000,9 \%$ in 2001 and $2 \%$ in 2002) [14]. Similarly, Lee, et al., who randomized patients into mandatory or selective CT groups, found the overall negative appendectomy rate to be significantly lower in the mandatory group $(2.6 \%$ versus $11.3 \%, 95 \% \mathrm{CI}=-3.5,26.3)$ [10]. Unique to our series however, was that the negative appendectomy rate did not differ significantly by gender (Fishers exact test, $\mathrm{p}=0.491)$. No difference was noted in the overall negative appendectomy rate by gender in the CT (Fishers exact test, $\mathrm{p}=1.00$ ) or non-CT group (Fishers exact test, $\mathrm{p}=0.75)$, and similarly no difference was noted by age $(\mathrm{CT} \mathrm{p}=0.14$, non-CT $\mathrm{p}=0.26)$. This is in contrast to many studies which fail to categorize patients as homogenous, receiving uniform benefit from CT regardless of age or gender. DeArmond, et al. compared negative appendectomy rates before and after the ready availability of CT at his institution [2]. Data revealed a lower overall negative appendectomy rate in the CT group $(14 \%$ vs $7 \%, \mathrm{p}<0.005)$, a benefit statistically significant only in males ages 13 to $50(11 \%$ vs $3 \%$, $\mathrm{p}<0.01)[2]$. A trend towards improved negative appendectomy rates in females ages 13 to 50 was noted (23\% versus $14 \%, \mathrm{p}=0.07)$, but did not reach statistical significance [2]. McCory, et al. who did not find CT to influence the overall ( $8 \%$ vs $9.4 \%, \mathrm{p}=0.36)$ or male $(6.1$ vs $6.0, \mathrm{p}=0.84)$ negative appendectomy rate, found the utilization of CT improved the negative appendectomy rates only in females $(13.7 \%$ vs $10.2 \%, \mathrm{p}=0.02$ ), more specifically those ages less than 5 $(0 \%$ vs $15 \%, \mathrm{p}=0.003)$ and greater than $45(9.3 \%$ vs $15 \%$, $\mathrm{p}=0.001$ ) [3]. Contrary to these reports, our data supports the routine use of $\mathrm{CT}$ in all patients regardless of age or gender.

Concerns have been raised regarding the additional time required to obtain a $\mathrm{CT}$ and whether it could negatively impact outcomes by delaying definitive management or imposing risks inherent to $\mathrm{CT}$ such as contrast related allergic reactions or oral contrast aspiration during anesthetic induction. Jones, et al., found that despite the increase use of $\mathrm{CT}$, average emergency room work up time was not significantly greater and the perforation rate declined from $25 \%$ to $9 \%$ [14]. Frei, et al., who investigated the consequences of increased CT utilization in the work up of acute appendicitis during a seven year period (12.3\% in 1998 to $84.4 \%$ in 2004 , $\mathrm{p}=0.001)$, found that the mean time to surgery was statistically greater $(\mathrm{p}=0.001)$ during the last four years as compared to the first three [19]. Despite the longer time to surgery, a significant decline in the overall complication rate, defined as perforations, gangrene, or abscess, was noted (33\% in 1998 vs $21.3 \%$ in $2004, \mathrm{p}=0.002$ ) [19]. While in our series the mean time to complete a CT was 4.3 hours, we do not know whether or not it was associated with increased 
emergency room work up times or longer times to surgery. We can however ascertain from our data that although not statistically significant, our CT group experienced a lower perforation rate than its non-CT counterpart $(9.5 \%$ vs $12.6 \%$, $\mathrm{p}=0.33)$, and the rate of gangrenous $(8 \%$ vs $5.5 \%, \mathrm{p}=0.47)$ and acute appendicitis with abscess $(1.3 \%$ vs $0 \%, \mathrm{p}=0.37)$ were also not found to be significantly different. Furthermore, the length of stay of the CT and non-CT group were similar (3.67 vs 3.63 days, $\mathrm{p}=0.92)$ making us strongly suspect that the additional CT related 4.3 hours did not negatively impact outcomes. In addition, although not reported in our statistics, we did not identify any $\mathrm{CT}$ related adverse reactions.

Eight hundred thirteen CT scans where obtained during the work up 940 patients who underwent surgery for acute appendicitis. Of the CT scans obtained, the sensitivity, specificity, negative predictive value, and positive predictive value was $99 \%, 61 \%, 68 \%$, and $99 \%$, with an overall accuracy of $98 \%$. These findings are comparable with those reported by earlier series $[3,4,7]$. Because our cohort only includes patients who underwent surgery we concede to the possibility that we potentially underestimate our false negative rate by excluding patients with negative CT scans who where erroneously discharged home. However, given the known validity of CT, our institutions CT protocol established to limit non-diagnostic studies, twenty-four hour radiology attending availability, and the mandatory examination of all patients presenting with abdominal pain by a senior surgical resident, we believe very few patients, if any at all presenting with appendicitis, would not have been captured.

\section{CONCLUSIONS}

The use of CT in the work up of acute appendicitis is associated with decreased negative appendectomy rates regardless of age and gender, and without any obvious untowardly affect on outcomes. In light of this we recommend that whenever possible a CT should be obtained for all patients presenting with abdominal pain in whom the diagnosis of acute appendicitis is considered. Traditional teachings tolerating $15 \%$ to $20 \%$ negative appendectomy rates should be abandoned, and 4\% adopted as a more acceptable standard. A large, randomized controlled study may need to be conducted in order to either validate our recommended mandatory approach or justify one which is more selective. In addition, cost analysis might be necessary to decide upon the necessity of performing a CT scan prior to appendectomy.

\section{REFERENCES}

[1] Flum DR, Koepsell T. The clinical and economic correlates of misdiagnosed appendicitis: nationwide analysis. Arch Surg 2002; 137(7): 799-804

[2] DeArmond GM, Dent DL, Myers JG, et al. Appendicitis: selective use of abdominal $\mathrm{CT}$ reduces negative appendectomy rate. Surg Infect 2003; 4(2): 213-8.

[3] McGory ML, Zingmond DS, Nanayakkara D, Maggard MA, Ko CY. Negative appendectomy rate: influence of CT scans. Am Surg 2005; 71(10): 803-8.

[4] Balthazar EJ, Rofsky NM, Zucker R. Appendicitis: the impact of computed tomography imaging on negative appendectomy and perforation rates. Am J Gastroenterol 1998; 93(5): 768-71.

[5] Ceydeli A, Lavotshkin S, Yu J, Wise L. When should we order a CT scan and when should we rely on the results to diagnose an acute appendicitis? Curr Surg 2006; 63(6): 464-8.

[6] Hershko DD, Sroka G, Bahouth H, Ghersin E, Mahajna A, Krausz MM. The role of selective computed tomography in the diagnosis and management of suspected acute appendicitis. Am Surg 2002; 68(11): 1003-7.

[7] Paulson EK, Kalady MF, Pappas TN. Clinical practice, suspected appendicitis. N Engl J Med 2003; 348(3): 236-42.

[8] Walker S, Haun W, Clark J, McMillin K, Zeren F, Gilliland T. The value of limited computed tomography with rectal contrast in the diagnosis of acute appendicitis. Am J Surg 2000; 180: 450-4.

[9] Neumayer L, Kennedy A. Imaging in appendicitis: a review with special emphasis on the treatment of women. Obstet Gynecol 2003; 102(6): 1404-9.

[10] Lee CC, Golub R, Singer AJ, Cantu R, Levinson H. Routine versus selective abdominal computed tomography scan in the evaluation of right lower quadrant pain: a randomized controlled trial. Acad Emerg Med 2007; 14: 117-22.

[11] Rao PM, Rhea JT, Novelline RA, Mostafavi AA, McCabe CJ. Effect of computed tomography of the appendix on treatment of patients and use of hospital resources. N Engl J Med 1998; 338: 1416.

[12] Kim K, Lee CC, Song KJ, Kim W, Suh G, Singer AJ. The impact of helical computed tomography on the negative appendectomy rate: a multi-center comparison. J Emerg Med 2008; 34(1): 3-6.

[13] Guss DA, Behling CA, Munassi D. Impact of abdominal helical computed tomography on the rate of negative appendicitis. J Emerg Med 2008; 34(1): 7-11.

[14] Jones K, Peña AA, Dunn EL, Nadalo L, Mangram AJ. Are negative appendectomies still acceptable? Am J Surg 2004; 188(6): 748-54.

[15] Rao PM, Rhea JT, Rattner DW, Venus LG, Novelline RA. Introduction of appendiceal CT: impact on negative appendectomy and appendiceal perforation rates. Ann Surg 1998; 229(3): 344-9.

[16] Musunuru S, Chen H, Rikkers LF, Weber SM. Computed tomography in the diagnosis of acute appendicitis: definitive or detrimental? J Gastrointest Surg 2007; 11(11): 1417-21.

[17] Lee SL, Walsh AJ, Ho HS. Computed tomography and ultrasonography do not improve and may delay the diagnosis and treatment of acute appendicitis. Arch Surg 2001; 136(5): 556-62.

[18] Vadeboncoeur TF, Heister RR, Behling CA, Guss DA. Impact of helical computed tomography on the rate of negative appendicitis. Am J Emerg Med 2006; 24(1): 43-7.

[19] Frei SP, Bond WF, Bazuro RK, Richardson DM, Sierzega GM, Reed JF. Appendicitis outcomes with increasing computed tomographic scanning. Am J Emerg Med 2008; 26(1): 39-44. 